\title{
Herbicidas alternativos no controle de Bidens pilosa e Euphorbia heterophylla resistentes a inibidores de ALS na cultura do algodão ${ }^{1}$
}

\author{
Alternative herbicides for controlling Bidens pilosa and Euphorbia heterophylla \\ resistant to ALS inhibitor in cotton crop
}

Guilherme Braga Pereira Braz²; Rubem Silvério de Oliveira Jr. ${ }^{3}$; Jamil Constantin³ ${ }^{3}$ Hugo de Almeida Dan ${ }^{4}$; Antonio Mendes de Oliveira Neto ${ }^{4}$; Gizelly Santos ${ }^{4}$; Alessandra Francischini Constantin $^{4}$; Hudson Kagueyama Takano

Resumo - A presença de Bidens pilosa e Euphorbia heterophylla resistentes aos herbicidas inibidores da ALS é um entrave no manejo de plantas daninhas na cultura do algodão, em função da escassez de alternativas de latifolicidas seletivos. Com o intuito de suprir essa carência de informações, foram instalados quatro experimentos em casa-de-vegetação com o objetivo de avaliar herbicidas aplicados em pós-emergência, recomendados para o algodão convencional e transgênico (resistente ao glyphosate e amonio-glufosinate), no controle destes biótipos resistentes. O delineamento experimental utilizado foi inteiramente casualizado com quatro repetições, sendo avaliados 21 tratamentos herbicidas, além de uma testemunha sem aplicação. Instalaram-se dois experimentos por espécie, onde o primeiro correspondeu à aplicação dos herbicidas em estádios de duas a quatro folhas e o segundo de quatro a seis. Avaliou-se os percentuais de controle aos 7 e 28 dias após a aplicação dos herbicidas. Amônio-glufosinate e glyphosate aplicados isolados ou em associações a outros herbicidas foram eficientes no controle de B. pilosa independente do estádio avaliado. Os dois biótipos demonstraram-se altamente resistente aos herbicidas inibidores de ALS avaliados. E. heterophylla demonstrou maior sensibilidade aos herbicidas amonio-glufosinate e glyphosate em aplicações precoces. Glyphosate destacou-se como boa alternativa no controle de E. heterophylla. Para este biótipo, foi verificado efeito sinérgico para as associações entre amonio-glufosinate e pyrithiobac-sodium.

Palavras-chave: Picão-preto, leiteiro, acetolactato sintase.

Abstract - The presence of Bidens pilosa and Euphorbia heterophylla resistant to ALS inhibitors is a problem to weed management in cotton crop, due to the fact that few alternatives broadleaf selective herbicides area available. In order to supply this information gap, four experiments were established in greenhouse conditions with the objective of evaluating herbicides applied in post-emergence conditions, recommended either for conventional and

\footnotetext{
${ }^{1}$ Recebido para publicação em 30/03/2011 e aceito em 08/06/2011.

2 Mestrando do Programa de Pós-Graduação em Agronomia da Universidade Estadual de Maringá (UEM/NAPD), Av. Colombo 5790, 87020-900, Maringá, PR. Email: <guilhermebrag@gmail.com>;

${ }^{3}$ Professores do Departamento de Agronomia da Universidade Estadual de Maringá (UEM);

${ }_{5}^{4}$ Alunos do Programa de Pós-Graduação em Agronomia da Universidade Estadual de Maringá (UEM);

${ }^{5}$ Acadêmicos de Agronomia da Universidade Estadual de Maringá (UEM).
} 
Braz et al.

transgenic cotton (resistant to glyphosate and amonio-glufosinate) for controlling these resistant biotypes. The experimental design was entirely randomized with four replications and 21 herbicides treatments, besides a check without herbicide application. Two experiments were conducted per species, where the first one corresponded to herbicides application in stages of two to four leaves, and the second in the stage of four to six. It was evaluated the percentage of control at 7 and 28 days after herbicide application. Amonio-glufosinate and glyphosate applied isolated or in combinations with other herbicides were effective in controlling B. pilosa independently of the growth stage. The two biotypes demonstrated to be highly resistant to ALS inhibitor herbicides. E. heterophylla showed greater sensitivity to herbicide amonio-glufosinate herbicide and glyphosate in early stages applications. Glyphosate stood out as a satisfactory alternative for controlling E. heterophylla. For this biotype, synergistic effect was verified for associations between amonio-glufosinate and pyrithiobac-sodium.

Keywords: beggar ticks, wild poinsettia, acetolactato sintase.

\section{Introdução}

O uso contínuo de herbicidas que possuem o mesmo mecanismo de ação seleciona plantas daninhas mais tolerantes, fazendo com que haja a seleção de biótipos resistentes. É importante destacar que os herbicidas não possuem capacidade de proporcionar mutação genética nas plantas. Entretanto, a pressão de seleção através da utilização intensiva de herbicidas faz com que as plantas demonstrem habilidades já adquiridas previamente em seu material genético, de sobreviver às condições adversas criadas pela utilização destes fitossanitários (Lamego et al., 2006).

Nas últimas décadas, foram relatadas diversas plantas daninhas resistentes à herbicidas no Brasil, sendo que o mecanismo de ação ao qual se registrou maior número de casos de resistência foi os herbicidas inibidores da ALS (Agostinetto \& Vargas, 2009). Dentre as espécies resistentes a este mecanismo de ação, Bidens pilosa e Euphorbia heterophylla consistem nos casos mais preocupantes em função da grande expansão territorial que os biótipos resistentes encontram-se disseminados e do elevado número de culturas que infestam (Hernandes et al., 2005; Trezzi et al., 2009).

As espécies B. pilosa e E. heterophylla são comumente encontradas infestando cultivos de algodão, sendo que a presença destas causam sérios prejuízos à cultura, reduzindo não somente a produtividade mas também a qualidade da fỉbra (Beltrão, 2004). Outro agravante da competição com estas espécies está relacionado aos fatores da fisiologia do algodoeiro, pois esta cultura possui metabolismo fotossintético C3 e taxa de crescimento inicial lenta (Freitas et al., 2002). Estas características desfavorecem a habilidade do algodão em competir pelos recursos necessários ao desenvolvimento com $B$. pilosa e E. heterophylla, que possuem rápido crescimento inicial.

A presença de biótipos resistentes a ALS nos cultivos de algodão convencional agravou-se em função das poucas alternativas herbicidas registradas para controle de folhas largas em pós-emergência. As únicas alternativas são os herbicidas trifloxysulfuronsodium e pyrithiobac-sodium, também inibidores da acetolactato sintase (Freitas et al., 2006).

Com a escassez de herbicidas registrados para o controle de folhas largas no algodão, as empresas direcionaram suas pesquisas para o desenvolvimento de genótipos de algodão que apresentem tolerância a aplicação de pós-emergentes, por meio de métodos biotecnológicos. Nos últimos anos 
Braz et al.

dois destes eventos biotecnológicos chegaram ao mercado brasileiro, os cultivares de algodão $\mathrm{LL}^{\circledR}$ e $\mathrm{RR}^{\circledR}$ que apresentam tolerância aos herbicidas amonio-glufosinate e glyphosate, respectivamente (Monquero, 2005).

Estes herbicidas possuem mecanismo de ação distinto aos produtos já registrados para utilização em pós-emergência no algodoeiro, e desta forma podem ser consideradas alternativas eficazes para ampliação do espectro de controle de plantas daninhas nesta cultura. Além disso, podem servir para evitar que haja a seleção de novos biótipos resistentes, sendo também ferramenta fundamental no controle de espécies já resistentes (Oliveira Neto et al., 2010).

Neste contexto, procurou-se no presente trabalho, encontrar alternativas eficazes no controle de $B$. pilosa e E. heterophylla resistentes a ALS, por meio da utilização de herbicidas pós-emergentes registrados para os cultivos de algodão convencional e/ou transgênico, sendo estes aplicados isoladamente e em associações.

\section{Material e métodos}

Os experimentos foram conduzidos em casa de vegetação no Centro de Treinamento em Irrigação (CTI), que pertence ao Campus sede da Universidade Estadual de Maringá (UEM), localizada em Maringá, PR. O período de condução dos ensaios foi de 28/09/2010 a $15 / 12 / 2010$.

O delineamento experimental utilizado foi inteiramente casualizado, com quatro repetições. Foram avaliados 21 tratamentos herbicidas aplicados em pós-emergência sobre dois estádios fenológicos ( 2 a 4; e 4 a 6 folhas) distintos de picão-preto (B. pilosa) e leiteiro $(E$. heterophylla), além de uma testemunha sem aplicação. Os tratamentos adotados com suas respectivas dosagens estão representados na Tabela 1.
As unidades experimentais foram compostas por vasos de $3 \mathrm{dm}^{3}$ de capacidade, os quais foram preenchidos com solo que apresentava valores de $\mathrm{pH}$ em água de 6,30; 2,94 $\mathrm{cmol}_{\mathrm{c}}$ de $\mathrm{H}^{+}+\mathrm{Al}^{+3} \mathrm{dm}^{-3}$ de solo; 5,30 $\mathrm{cmol}_{\mathrm{c}} \mathrm{dm}^{-3}$ de $\mathrm{Ca}^{+2} ; 1,56 \mathrm{cmol}_{\mathrm{c}} \mathrm{dm}^{-3}$ de $\mathrm{Mg}^{+2}$; $0,37 \mathrm{cmol}_{\mathrm{c}} \mathrm{dm}^{-3}$ de $\mathrm{K}^{+} ; 4,40 \mathrm{mg} \mathrm{dm}^{-3}$ de P; 7,90 $\mathrm{g} \mathrm{dm}^{-3}$ de C; $250 \mathrm{~g} \mathrm{~kg}^{-1}$ de areia grossa; $260 \mathrm{~g}$ $\mathrm{kg}^{-1}$ de areia fina; $20 \mathrm{~g} \mathrm{~kg}^{-1}$ de silte e $470 \mathrm{~g} \mathrm{~kg}^{-1}$ de argila.

Antecedendo a semeadura das plantas daninhas os vasos foram irrigados, sendo semeadas quarenta sementes de B. pilosa resistente a ALS, por vaso; para a espécie $E$. heterophylla, o número de sementes foi trinta. As sementes de $B$. pilosa resistentes foram oriundas de plantas do ensaio realizado por Guerra et al. (2011), no qual os autores comprovaram resistência deste biótipo aos herbicidas inibidores da ALS. As sementes de E. heterophylla foram oriundas de áreas do estado do Paraná com histórico de resistência a inibidores de ALS já relatado (Heap, 2011).

As plantas emergidas após a aplicação dos herbicidas foram retiradas das unidades experimentais, a fim de que o controle fosse baseado apenas nas plantas que receberam a aplicação dos herbicidas em pós-emergência. Durante a condução do ensaio, realizou-se a irrigação diária dos vasos, por meio de sistema automatizado.

Para todas as aplicações foi utilizado um pulverizador costal de pressão constante à base de $\mathrm{CO}_{2}$, equipado com barra munida de três pontas tipo jato leque XR 110.02, espaçadas de $50 \mathrm{~cm}$ entre si, sob pressão de 2,0 $\mathrm{kgf} \mathrm{cm}^{-2}$. Estas condições de aplicação proporcionaram o equivalente a $200 \mathrm{~L} \mathrm{ha}^{-1}$ de calda. No momento da aplicação do experimento (E1), as condições climáticas encontradas foram: Temp. $=28^{\circ} \mathrm{C}$; UR $=55 \%$; velocidade do vento $=2 \mathrm{~km} \mathrm{~h}^{-1}$. As condições climáticas encontradas na aplicação do segundo experimento (E2) foram: Temp. = 
Braz et al.

$24,5^{\circ} \mathrm{C}, \mathrm{UR}=83 \%$, velocidade do vento $=2,5$

$\mathrm{km} \mathrm{h}^{-1}$.

Tabela 01. Relação dos herbicidas e respectivas doses utilizadas nos experimentos de controle de B. pilosa e E. heterophylla resistentes aos inibidores de ALS. Maringá, 2010.

\begin{tabular}{lc}
\hline \multicolumn{1}{c}{ Tratamentos } & Dosagem $\left(\mathbf{g ~ h a} \mathbf{~}^{-1}\right)$ \\
\hline 01. pyrithiobac-sodium & 16,8 \\
02. pyrithiobac-sodium & 28 \\
03. pyrithiobac-sodium & 56 \\
04. pyrithiobac-sodium & 84 \\
05. amonio-glufosinate & 300 \\
06. amonio-glufosinate & 400 \\
07. amonio-glufosinate & 500 \\
08. glyphosate & 648 \\
09. glyphosate & 972 \\
10. amonio-glufosinate + pyrithiobac-sodium & $300+16,8$ \\
11. amonio-glufosinate + pyrithiobac-sodium & $300+28$ \\
12. amonio-glufosinate + pyrithiobac-sodium & $300+56$ \\
13. amonio-glufosinate + pyrithiobac-sodium & $400+16,8$ \\
14. amonio-glufosinate + pyrithiobac-sodium & $400+28$ \\
15. amonio-glufosinate + pyrithiobac-sodium & $400+56$ \\
16. glyphosate + pyrithiobac-sodium & $648+16,8$ \\
17. glyphosate + pyrithiobac-sodium & $648+28$ \\
18. glyphosate + pyrithiobac-sodium & $648+56$ \\
19. trifloxysulfuron-sodium & 3 \\
20. trifloxysulfuron-sodium + pyrithiobac-sodium & $2,25+16,8$ \\
21. trifloxysulfuron-sodium + pyrithiobac-sodium & $2,25+42$ \\
22. Testemunha sem herbicida & - \\
\hline
\end{tabular}

As avaliações realizadas foram: Resultados e discussão porcentagem de controle aos 7 e 28 dias após a aplicação (DAA), usando uma escala onde $0 \%$ corresponde à ausência de injúrias e $100 \%$ à morte das plantas, de acordo com recomendações da Sociedade Brasileira da Ciência das Plantas Daninhas (SBCPD, 1995).

Após serem tabulados, os dados foram submetidos à análise de variância pelo teste $\mathrm{F}$, e quando se verificou efeito significativo para alguma variável-resposta, as médias foram comparadas pelo teste de agrupamento de Scott-Knott ao nível de $5 \%$ de probabilidade.

A aplicação de amonio-glufosinate, isolado ou em mistura com pyrithiobac-sodium ou glyphosate (972 $\mathrm{g} \mathrm{ha}^{-1}$ ), sobre plantas de $B$. pilosa com duas a quatro folhas causaram maiores níveis de controle aos 7 DAA (Tabela 02). Destaca-se que a associação entre estes herbicidas não apresentou efeito antagônico, o que possibilita a utilização destes em conjunto. Além disso, a atividade residual de pyrithiobac-sodium pode ser considerada uma boa alternativa no manejo de outras plantas daninhas (Koger et al., 2007).

O herbicida glyphosate nas dosagens de 648 e $972 \mathrm{~g} \mathrm{ha}^{-1}$, e as suas associações com 
Braz et al.

pyrithiobac-sodium também apresentaram bons níveis de controle sobre $B$. pilosa. Tanto pyrithiobac-sodium quanto trifloxysulfuronsodium causaram efeitos supressivos sobre a espécie em estudo, entretanto, estes percentuais foram insatisfatórios, comprovando a alta resistência que este biótipo apresenta aos herbicidas inibidores da ALS. Nicolai et al. (2006) verificaram para os herbicidas imazethapyr, chlorimuron-methyl e nicosulfuron níveis de supressão acima de 50\% sobre B. pilosa resistente ALS.

Tabela 02. Porcentagens de controle de B. pilosa (E1) aos 7 e 28 DAA, em função da aplicação de diferentes herbicidas utilizados em pós-emergência do algodoeiro Maringá, 2010.

\section{Tratamentos (Dosagem: $\mathrm{g} \mathrm{ha}^{-1}$ )}

01. pyrithiobac-sodium $(16,8)$

02. pyrithiobac-sodium (28)

03. pyrithiobac-sodium (56)

04. pyrithiobac-sodium (84)

05. amonio-glufosinate (300)

06. amonio-glufosinate (400)

07. amonio-glufosinate (500)

08. glyphosate (648)

09. glyphosate (972)

10. amonio-glufosinate + pyrithiobac-sodium $(300+16,8)$

11. amonio-glufosinate + pyrithiobac-sodium $(300+28)$

12. amonio-glufosinate + pyrithiobac-sodium $(300+56)$

13. amonio-glufosinate + pyrithiobac-sodium $(400+16,8)$

14. amonio-glufosinate + pyrithiobac-sodium $(400+28)$

15. amonio-glufosinate + pyrithiobac-sodium $(400+56)$

16. glyphosate + pyrithiobac-sodium $(648+16,8)$

17. glyphosate + pyrithiobac-sodium $(648+28)$

18. glyphosate + pyrithiobac-sodium $(648+56)$

19. trifloxysulfuron-sodium (3)

20. trifloxysulfuron-sodium + pyrithiobac-sodium $(2,25+16,8)$

21. trifloxysulfuron-sodium + pyrithiobac-sodium $(2,25+42)$

22. Test. sem herbicida

$\mathrm{CV}(\%)$

DAA: Dias após a aplicação; Médias seguidas de mesmas letras na coluna não diferem entre si pelo teste de Scott-knott $(p \leq 0,05)$.

$\mathrm{Na}$ última avaliação de controle (28 DAA), nota-se que os tratamentos a base de amonio-glufosinate tiveram grande eficácia quando aplicado em estádios mais precoces de desenvolvimento de $B$. pilosa; os resultados observados para glyphosate foram semelhantes. A aplicação de dosagens superiores a $600 \mathrm{~g}$ ha
${ }^{1}$ de glyphosate sobre $B$. pilosa, no estádio de duas a quatro folhas, já foi relatada por ser altamente eficaz (Nicolai et al., 2006).

Os herbicidas inibidores da ALS avaliados não apresentaram efeito algum sobre B. pilosa aos 28 DAA, havendo clorose nas plantas apenas na primeira avaliação de 
Braz et al.

controle. A resistência aos inibidores da enzima ALS, em geral, é considerada de elevado grau, sendo que as espécies demonstram poucos sintomas de intoxicação por estes herbicidas. Isto advém em função da resistência ocorrer em uma alteração da suscetibilidade da enzima, fazendo com que a planta se torne altamente tolerante ao sítio de ação do herbicida (López-Ovejero et al., 2006).

Ressalta-se ainda, que a resistência apresentada por $B$. pilosa é cruzada, o que faz com que este biótipo demonstre-se resistente à aplicação de inibidores da ALS, independente do grupo químico ao qual o herbicida pertence. A resistência cruzada apresentada por esta espécie já foi comprovada em outros trabalhos, onde foi verificado que nenhum dos quatro grupos químicos, que possuem como mecanismo de ação a inibição da enzima ALS, possuem eficiência no controle deste biótipo resistente (Christoffoleti, 2002).

O desempenho do amonio-glufosinate no controle de $B$. pilosa, quando as plantas encontravam-se mais desenvolvidas (quatro a seis folhas), foi semelhante ao observado no primeiro estádio. Para este, também não houve antagonismo nas misturas com pyrithiobacsodium e a aplicação amonio-glufosinate isolado foi eficaz, aos 7 DAA (Tabela 03).
O glyphosate $\left(648 \mathrm{~g} \mathrm{ha}^{-1}\right)$ isolado e em mistura com pyrithiobac-sodium exerceu controle regular de aproximadamente $75 \%$, não atingindo percentuais de controle satisfatórios na primeira avaliação $(>80 \%)$. Em contrapartida, quando se incrementou a dosagem de glyphosate para $972 \mathrm{~g} \mathrm{ha}^{-1} \mathrm{a}$ performance obtida foi equivalente a dos tratamentos a base de amonio-glufosinate.

$\mathrm{Na}$ última avaliação de controle verificou-se morte de todas as plantas de $B$. pilosa (E2), quando submetidas à aplicação de amonio-glufosinate isolado ou em combinação com diferentes dosagens de pyrithiobacsodium. A aplicação de glyphosate isolado e em mistura ao herbicida pyrithiobac-sodium também apresentou elevada eficácia no controle desta espécie.

A alta sensibilidade que esta espécie apresentou ao glyphosate e amonio-glufosinate, aliada a inexistência de efeitos antagônicos da associação destes com o pyrithiobac-sodium, indica duas importantes ferramentas no manejo de populações de Bidens pilosa resistente em lavouras de algodão. Em áreas com grande infestação desta espécie, o produtor pode optar por cultivares geneticamente modificadas a fim de garantir o manejo desta espécie. 
Braz et al.

Tabela 03. Porcentagens de controle de B. pilosa (E2) aos 7 e 28 DAA, em função da aplicação de diferentes herbicidas utilizados em pós-emergência do algodoeiro Maringá, 2010.

Tratamentos (Dosagem: $\mathrm{g} \mathrm{ha}^{-\mathbf{1}}$ )

01. pyrithiobac-sodium $(16,8)$

02. pyrithiobac-sodium (28)

03. pyrithiobac-sodium (56)

04. pyrithiobac-sodium (84)

05. amonio-glufosinate (300)

06. amonio-glufosinate (400)

07. amonio-glufosinate (500)

08. glyphosate (648)

09. glyphosate (972)

10. amonio-glufosinate + pyrithiobac-sodium $(300+16,8)$

11. amonio-glufosinate + pyrithiobac-sodium $(300+28)$

12. amonio-glufosinate + pyrithiobac-sodium $(300+56)$

13. amonio-glufosinate + pyrithiobac-sodium $(400+16,8)$

14. amonio-glufosinate + pyrithiobac-sodium $(400+28)$

15. amonio-glufosinate + pyrithiobac-sodium $(400+56)$

16. glyphosate + pyrithiobac-sodium $(648+16,8)$

17. glyphosate + pyrithiobac-sodium $(648+28)$

18. glyphosate + pyrithiobac-sodium $(648+56)$

19. trifloxysulfuron-sodium (3)

20. trifloxysulfuron-sodium + pyrithiobac-sodium $(2,25+16,8)$

21. trifloxysulfuron-sodium + pyrithiobac-sodium $(2,25+42)$

22. Test. sem herbicida
Controle (\%)

7 DAA 28 DAA

$0 \mathrm{~d}$

$0 \mathrm{~d}$

$0 \mathrm{~d}$

$0 \mathrm{~d}$

99 a

99 a

98 a

$75 \mathrm{~b}$

96 a

99 a

99 a

99 a

99 a

99 a

99 a

77 b

77 b

$70 \mathrm{c}$

$0 \mathrm{~d}$

$0 \mathrm{~d}$

$0 \mathrm{~d}$

$0 \mathrm{~d}$

4,35
$0 \mathrm{c}$

0 c

$0 \mathrm{c}$

$0 \mathrm{c}$

$100 \mathrm{a}$

$100 \mathrm{a}$

100 a

100 a

$100 \mathrm{a}$

100 a

$100 \mathrm{a}$

$100 \mathrm{a}$

100 a

$100 \mathrm{a}$

100 a

$98 \mathrm{~b}$

$98 \mathrm{~b}$

$100 \mathrm{a}$

$0 \mathrm{c}$

$0 \mathrm{c}$

$0 \mathrm{c}$

0 c

\begin{tabular}{lrc}
\hline $\mathrm{CV}(\%)$ & 4,35 & 1,86 \\
\hline
\end{tabular}

"DAA: Dias após a aplicação; Médias seguidas de mesmas letras na coluna não diferem entre si pelo teste de Scott-knott $(p \leq 0,05)$. 
Braz et al.

Tabela 04. Porcentagens de controle de E. heterophylla (E1) aos 7 e 28 DAA, em função da aplicação de diferentes herbicidas utilizados em pós-emergência do algodoeiro Maringá, 2010.

\section{Tratamentos (Dosagem: $\mathrm{g} \mathrm{ha}^{-1}$ )}

01. pyrithiobac-sodium $(16,8)$

02. pyrithiobac-sodium (28)

03. pyrithiobac-sodium (56)

04. pyrithiobac-sodium (84)

05. amonio-glufosinate (300)

06. amonio-glufosinate (400)

07. amonio-glufosinate (500)

08. glyphosate (648)

09. glyphosate (972)

10. amonio-glufosinate + pyrithiobac-sodium $(300+16,8)$

11. amonio-glufosinate + pyrithiobac-sodium $(300+28)$

12. amonio-glufosinate + pyrithiobac-sodium $(300+56)$

13. amonio-glufosinate + pyrithiobac-sodium $(400+16,8)$

14. amonio-glufosinate + pyrithiobac-sodium $(400+28)$

15. amonio-glufosinate + pyrithiobac-sodium $(400+56)$

16. glyphosate + pyrithiobac-sodium $(648+16,8)$

17. glyphosate + pyrithiobac-sodium $(648+28)$

18. glyphosate + pyrithiobac-sodium $(648+56)$

19. trifloxysulfuron-sodium (3)

20. trifloxysulfuron-sodium + pyrithiobac-sodium $(2,25+16,8)$

21. trifloxysulfuron-sodium + pyrithiobac-sodium $(2,25+42)$

22. Test. sem herbicida
Controle (\%)

7 DAA $^{*} 28$ DAA

$29 \mathrm{~d}$

$0 \mathrm{e}$

$20 \mathrm{e}$

$19 \mathrm{e}$

$33 \mathrm{~d}$

$75 \mathrm{~b}$

89 a

93 a

$80 \mathrm{~b}$

95 a

$80 \mathrm{~b}$

85 a

88 a

91 a

90 a

89 a

88 a

86 a

$82 \mathrm{~b}$

$30 \mathrm{~d}$

$40 \mathrm{c}$

$25 \mathrm{e}$

$0 \mathrm{f}$

8,87
$0 \mathrm{e}$

0 e

0 e

$76 \mathrm{~b}$

82 b

96 a

90 a

97 a

$69 \mathrm{~b}$

73 b

80 b

92 a

82 b

81 b

$100 \mathrm{a}$

93 a

96 a

$33 \mathrm{c}$

$20 \mathrm{~d}$

0 e

$0 \mathrm{e}$

"DAA: Dias após a aplicação; Médias seguidas de mesmas letras na coluna não diferem entre si pelo teste de Scott-knott $(p \leq 0,05)$.

Para E. heterophylla, em avaliação de controle realizada aos 7 DAA, verificou-se que o amonio-glufosinate $\left(300 \mathrm{~g} \quad \mathrm{ha}^{-1}\right)$ em associação ao pyrithiobac-sodium, em dosagem superior a $28 \mathrm{~g} \mathrm{ha}^{-1}$, e os demais tratamentos aplicação de amonio-glufosinate acima de 400 $\mathrm{g} \mathrm{ha}^{-1}$, isoladamente ou em mistura, apresentaram controle satisfatório desta espécie daninha (Tabela 04). Destacam-se também as aplicações com glyphosate, que proporcionaram níveis satisfatórios de controle, independentemente das dosagens e da mistura.

$\mathrm{Na}$ primeira avaliação de controle, a utilização isolada dos herbicidas inibidores da ALS (pyrithiobac-sodium e trifloxysulfuron- sodium) apresentou limitado efeito de supressão sobre E. heterophylla, indicando o elevado grau da resistência e a necessidade de outras alternativas de controle.

A última avaliação de controle realizada nas plantas de E. heterophylla, em estádio de duas a quatro folhas, demonstrou que o glyphosate apresenta alta eficiência sobre esta espécie, sendo que em todas as dosagens isoladas e em associação com pyrithiobacsodium, os percentuais de controle se mantiveram superiores à 90\%. A aplicação de amonio-glufosinate + pyrithiobac-sodium (300 + $56 \mathrm{~g} \mathrm{ha}^{-1}$ ) e amonio-glufosinate em dosagem superior a $400 \mathrm{~g} \mathrm{ha}^{-1}$, isoladamente e em 
Braz et al.

associação a pyrithiobac-sodium, também apresentaram níveis de controle satisfatórios.

O pyrithiobac-sodium isolado não apresentou controle sobre E. heterophylla aos 28 DAA. Entretanto, para o outro herbicida que pertencente ao grupo químico das sulfoniluréias (trifloxysulfuron-sodium) verificou-se que a sua aplicação isolada causou leve clorose foliar, todavia, os níveis de controle foram muito baixos. Esta diferença de plantas resistentes na sensibilidade a herbicidas de mesmo mecanismo de ação, pode estar relacionada ao fato de estes herbicidas serem de grupos químicos distintos.

Os percentuais de controle aos 7 DAA sob as plantas de E. heterophylla, em estádio de quatro a seis folhas, foram semelhantes aos observados nas plantas que receberam os mesmos herbicidas em aplicações mais precoces (Tabela 05). A utilização de amonioglufosinate isolado ou em mistura com pyrithiobac-sodium apresentou maiores níveis de controle, exceto nos tratamentos com amonio-glufosinate $300,0 \mathrm{~g} \mathrm{ha}^{-1}$ isolado ou associado ao pyrithiobac-sodium $\left(28 \mathrm{~g} \mathrm{ha}^{-1}\right)$.

Entre os tratamentos que tiveram como base o herbicida glyphosate, apenas a maior dosagem foi capaz de igualar o desempenho dos tratamentos com amonioglufosinate no controle de E. heterophylla, fato esse observado na primeira avaliação (7 DAA). Em geral, o incremento de dosagem do glyphosate proporcionou maiores níveis de controle. Isto se deve em parte ao mecanismo de ação que ele apresenta, fazendo com que a capacidade de metabolização deste herbicida seja reduzida com o aumento da dosagem (Correia \& Durigan, 2010).

Os herbicidas inibidores da ALS não apresentaram efeito de controle inicial sobre o leiteiro em estádio de crescimento mais avançado, verificando que as plantas que receberam a aplicação de pyrithiobac-sodium e trifloxysulfuron-sodium encontravam-se semelhantes à testemunha sem herbicida. Verificou-se que apenas as plantas de $E$. heterophylla que receberam aplicação de $400 \mathrm{~g}$ $\mathrm{ha}^{-1}$ de amonio-glufosinate em associação com pyrithiobac-sodium, em dosagens superior a 28 $\mathrm{g} \mathrm{ha}^{-1}$, apresentaram menor porcentagem de plantas com rebrotadas, indicando que esta mistura diminui a capacidade de recuperação desta espécie pelas injúrias provocadas pelos herbicidas.

Em função da velocidade de desencadeamento dos sintomas provocados pela aplicação do amonio-glufosinate, as folhas das plantas que receberam a aplicação deste herbicida tendem a senescer rapidamente; entretanto, algumas espécies possuem altos teores de carboidratos nos seus tecidos de reserva, fazendo com que estas plantas possuam alta capacidade de rebrotar (Carbonari et al., 2004). Além disso, a ação de contato que este produto possui faz com que não haja redistribuição deste para os outros órgãos das plantas; característica esta que permite as plantas rebrotarem, caso o herbicida não cause a morte das plantas tratadas (Oliveira Jr. et al., 2006). 
Braz et al.

Tabela 05. Porcentagens de controle de E. heterophylla (E2) aos 7 e 28 DAA, em função da aplicação de diferentes herbicidas utilizados em pós-emergência do algodoeiro Maringá, 2010.

Tratamentos (Dose: $\mathrm{g} \mathrm{ha}^{-\mathbf{1}}$ )

01. pyrithiobac-sodium $(16,8)$

02. pyrithiobac-sodium (28)

03. pyrithiobac-sodium (56)

04. pyrithiobac-sodium (84)

05. amonio-glufosinate (300)

06. amonio-glufosinate (400)

07. amonio-glufosinate (500)

08. glyphosate (648)

09. glyphosate (972)

10. amonio-glufosinate + pyrithiobac-sodium $(300+16,8)$

11. amonio-glufosinate + pyrithiobac-sodium $(300+28)$

12. amonio-glufosinate + pyrithiobac-sodium $(300+56)$

13. amonio-glufosinate + pyrithiobac-sodium $(400+16,8)$

14. amonio-glufosinate + pyrithiobac-sodium $(400+28)$

15. amonio-glufosinate + pyrithiobac-sodium $(400+56)$

16. glyphosate + pyrithiobac-sodium $(648+16,8)$

17. glyphosate + pyrithiobac-sodium $(648+28)$

18. glyphosate + pyrithiobac-sodium $(648+56)$

19. trifloxysulfuron-sodium (3)

20. trifloxysulfuron-sodium + pyrithiobac-sodium $(2,25+16,8)$

21. trifloxysulfuron-sodium + pyrithiobac-sodium $(2,25+42)$

22. Test. sem herbicida

$\mathrm{CV}(\%)$
Controle (\%)

7 DAA $^{*} \quad 28$ DAA

$0 \mathrm{c}$

$0 \mathrm{c}$

$0 \mathrm{c}$

$0 \mathrm{c}$

$73 \mathrm{~b}$

79 a

83 a

75 b

85 a

80 a

$73 \mathrm{~b}$

83 a

79 a

87 a

88 a

$72 \mathrm{~b}$

$67 \mathrm{~b}$

75 b

$0 \mathrm{c}$

$0 \mathrm{c}$

$0 \mathrm{c}$

$0 \mathrm{c}$

13,48
$0 \mathrm{~d}$

$0 \mathrm{~d}$

$0 \mathrm{~d}$

$0 \mathrm{~d}$

$31 \mathrm{c}$

$34 \mathrm{c}$

$21 \mathrm{c}$

83 a

100 a

$33 \mathrm{c}$

$54 \mathrm{~b}$

58 b

$41 \mathrm{c}$

80 a

83 a

78 a

71 B

72 b

$15 \mathrm{~d}$

$13 \mathrm{~d}$

$18 \mathrm{~d}$

$0 \mathrm{~d}$

"DAA: Dias após a aplicação; Médias seguidas de mesmas letras na coluna não diferem entre si pelo teste de Scottknott $(p \leq 0,05)$. 
Braz et al.

O glyphosate apresentou controle satisfatório nas duas dosagens utilizadas quando aplicado isoladamente, obtendo-se níveis de controle igual a $100 \%$ na maior dose deste herbicida. Ramires et al (2010) verificaram a mesma eficiência apresentada pelo glyphosate no controle de leiteiro em estádio de quatro a seis folhas. Corroborando com os resultados do estádio de aplicação mais precoce, o leiteiro resistente a ALS demonstrou ligeira sensibilidade ao trifloxysulfuron-sodium.

Em comparação com picão-preto, o leiteiro resistente a ALS ofereceu maior dificuldade de manejo por meio do controle químico. Em aplicações precoces (estádio de duas a quatro folhas) os biótipos de $B$. pilosa e E. heterophylla foram mais sensíveis aos herbicidas aplicados em pós-emergência.

\section{Conclusão}

As aplicações em pós-emergência nestas espécies em estádios mais precoces exerceu melhor controle quando comparada as tardias. $\mathrm{O}$ biótipo de $B$. pilosa resistente a inibidores da ALS possui alta suscetibilidade aos herbicidas amonio-glufosinate e glyphosate, sendo que a associação entre estes herbicidas com pyrithiobac-sodium não apresentou efeito antagônico.

A associação entre amonioglufosinate e pyrithiobac-sodium apresentou efeito sinérgico no controle de Euphorbia heterophylla resistente a ALS. Para este biótipo, a aplicação de glyphosate, isolado ou em associação, destacou-se como boa alternativa no controle de E. heterophylla.

\section{Referências}

AGOSTINETTO, A.; VARGAS, L. Resistência de plantas daninhas a herbicidas no Brasil. Passo Fundo: Gráfica Editora Berthier, 2009. 352p.

BELTRÃO, N.E.M. Manejo e controle de plantas daninhas em algodão. In: VARGAS, L.; ROMAN, E. S. (Eds.). Manual de manejo e controle de plantas daninhas. Bento Gonçalves: Embrapa Uva e Vinho, 2004. p.215-250.

CARBONARI, C.A. et al. Controle químico de Panicum repens e Paspalum repens. Planta Daninha, v.22, n.3, p.453-460, 2004.

CORREIA, N.M.; DURIGAN, J.C. Controle de plantas daninhas na cultura de soja resistente ao glyphosate. Bragantia, v.69, n.2, p.319-327, 2010.

CHRISTOFFOLETI, P.J. Curvas de doseresposta de biótipos resistente e suscetível de Bidens pilosa L. aos herbicidas inibidores da ALS. Scientia Agrícola, v.59, n.3, p.513519, 2002.

FREITAS, R.S. et al. Manejo de plantas daninhas na cultura do algodoeiro em sistema de plantio direto. Planta Daninha, v.24, n.2, p.339-346, 2006.

FREITAS, R.S. et al. Interferência de plantas daninhas na cultura de algodão em sistema de plantio direto. Planta Daninha, v.20, n.2, p.197-205, 2002.

GUERRA, N. et al. Efeito da modalidade de aplicação sobre o controle de Bidens pilosa resistente a herbicidas inibidores da enzima ALS. Global Science and Technology, v.4, n.1, p.61-69, 2011.

HEAP, I. International survey of resistant weeds. Disponível em: <http://www. weedsciencie.org/in.asp >. Acesso em: 5 junho 2011.

HERNANDES, G.C. et al. Levantamento de práticas agronômicas e distribuição geográfica de Bidens spp. resistentes aos herbicidas inibidores de ALS nos Estados do 
Braz et al.

Rio Grande do Sul e do Paraná. Planta Daninha, v.23, n.4, p.677-682, 2005.

KOGER, C.H. et al. Effect of residual herbicides used in the last post-directed application on weed control and cotton yield in glyphosate- and glufosinate-resistant cotton. Weed Technology, v.21, n.2, p.378383, 2007.

LAMEGO, F.P. et al. Distância genética e geográfica entre acessos de picão-preto suscetíveis e resistentes a herbicidas inibidores da acetolactato sintase. Pesquisa Agropecuária Brasileira, v.41, n.6, p.963968, 2006.

LÓPEZ-OVEJERO, R.F. et al. Resistance and differential susceptibility of Bidens pilosa and $B$. subalternans biotypes to ALSinhibiting herbicides. Scientia Agrícola, v.63, n.2, p.139-145, 2006.

MONQUERO, P.A. Plantas transgênicas resistentes aos herbicidas: situação e perspectivas. Bragantia, v.64, n.4, p.517531, 2005.

NICOLAI, M. et al. Alternativas de manejo para as populações de picão-preto (Bidens pilosa e Bidens subalternans) resistentes aos herbicidas inibidores da ALS. Revista Brasileira de Herbicidas, v.5, n.3, p.72-79, 2006.

OLIVEIRA NETO, A.M. et al. Estratégias de manejo de inverno e verão visando ao controle de Conyza bonariensis e Bidens pilosa. Planta Daninha, v.28, n.5, p.11071116, 2010.

OLIVEIRA JR., R.S. et al. Aplicações seqüenciais de flumiclorac-pentil para $o$ controle de Euphorbia heterophylla na cultura da soja. Acta Scientiarum Agronomy, v. 28, n.1, p.115-122, 2006

RAMIRES, A.C. et al. Controle de Euphorbia heterophylla e Ipomoea grandifolia com a utilização de glyphosate isolado ou em associação com latifolicidas. Planta Daninha, v.28, n.3, p.621-629, 2010.

SOCIEDADE BRASILEIRA DA CIÊNCIA DAS PLANTAS DANINHAS. Procedimentos para instalação, avaliação e análise de experimentos com herbicidas. Londrina: SBCPD, 1995. 42p.

TREZZI, M.M. et al. Características morfofisiológicas de biótipos de Euphorbia heterophylla com resistência a diferentes mecanismos de ação herbicida. Planta Daninha, v.27, n.5, p.1075-1082, 2009. 